\title{
Jejunal Ulcer, CTCAE
}

National Cancer Institute

\section{Source}

National Cancer Institute. Lejunal Ulcer, CT CAE. NCI Thesaurus. Code C57832.

A disorder characterized by a circumscribed, erosive lesion on the mucosal surface of the jejunum. 